\title{
Rectal Pain
}

National Cancer Institute

\section{Source}

National Cancer Institute. Rectal Pain. NCI Thesaurus. Code C78587.

Pain in the rectal area. 